Quim. Nova, Vol. 36, No. 3, 480-483, 2013

\title{
O TEMPO DE PEGA EM GELATINAS COMERCIAIS: UMA EXPERIÊNCIA DA DISCIPLINA DE QUIMIOMETRIA PARA ESTUDANTES DE GRADUAÇÃO EM QUÍMICA
}

\author{
Thiago Lucena de Macedo Guedes, Maurity Sanderson de Lima Soares, Luiz Seixas das Neves e Kássio Michell Gomes \\ de Lima* \\ Instituto de Química, Universidade Federal do Rio Grande do Norte, 59072-970 Natal - RN, Brasil
}

Recebido em 29/5/12; aceito em 5/10/12; publicado na web em 18/2/13

\begin{abstract}
INVESTIGATION OF THE SETTING-TIME OF COMMERCIAL GELATINS: AN EXPERIMENT FROM A CHEMOMETRIC COURSE FOR UNDERGRADUATE CHEMISTRY STUDENTS. The aim of this paper is to describe a chemometrics experiment for undergraduate chemistry students using basic concepts of experimental design to determine setting-time of commercial gelatins.
\end{abstract}

Keywords: experimental design; hardening; chemometrics course.

\section{INTRODUÇÃO}

A quimiometria, segundo a IUPAC, ${ }^{1}$ pode ser definida como a aplicação da estatística na análise de dados químicos e planejamento de experimentos químicos e simulações. A aplicação de métodos de calibração multivariada, técnicas de reconhecimento de padrão e planejamentos experimentais para resolução de problemas químicos são alguns dos temas muitas vezes relacionados à quimiometria.

Por outro lado, apesar do crescente uso da quimiometria para resolução de problemas em diversos campos da pesquisa, ${ }^{2,3}$ relativamente poucas experiências têm sido apresentadas sobre experimentos didáticos de quimiometria envolvendo alunos de graduação em Instituições de Ensino Superior (IES) brasileiras. Este artigo se propôs a descrever um experimento didático da disciplina de quimiometria do curso de graduação em Química da UFRN.

\section{APLICAÇÃO: TEMPO DE PEGA EM GELATINAS COMERCIAIS}

A gelatina é uma proteína resultante da degradação hidrolítica do colágeno e que não contém glúten, colesterol, gordura ou carboidratos em sua composição. Sua aplicação na indústria e na ciência é crescente, destacando-se os empregos nas indústrias alimentícia, farmacêutica, cosmética e fotográfica. O processo termo-reversível de gelação da gelatina em solução envolve a ordenação das moléculas de gelatina, de forma a tentar reorganizar a estrutura original do colágeno, formando tripla-hélices mantidas unidas por ligações de hidrogênio e que compõem agregados crescentes e interconectados até que todo o volume do sistema seja percolado e o ponto de gelação seja atingido. As propriedades da gelatina que mais chamam atenção para sua utilização são a força gel, as temperaturas de gelação ("pega") e de derretimento e a sua viscosidade. ${ }^{4,5}$

Algumas características devem ser observadas com relação ao processo de preparação da gelatina, que pode alterar drasticamente as características do produto obtido. Durante a dissolução, os fatores preponderantes serão a distribuição de tamanhos de partículas e a técnica de dispersão e mistura do pó em água. ${ }^{6,7}$ Em geral, uma quantidade adequada de água é empregada a temperaturas na faixa de 60 a $80{ }^{\circ} \mathrm{C}$ para a adição gradual do pó de gelatina. Dependendo da técnica (ou equipamento) de agitação empregada, uma série de problemas pode surgir, dentre os quais os mais importantes são a

*e-mail: kassio@ufrnet.br formação de agregados e espumas (que geralmente acarretam perda de força gel); normalmente isso é consequência de longos tempos de dissolução ou das altas energias mecânicas de agitação.

Um dos métodos empregados na caracterização de gelatinas comerciais é a determinação de força gel empregando o gelômetro de Bloom. Esse teste é conduzido em géis de concentração 6,67\% endurecidos sob resfriamento a $10{ }^{\circ} \mathrm{C}$ por 16 a $18 \mathrm{~h}$; a medida é feita como o peso mínimo necessário para deformar a superfície do sistema em $4 \mathrm{~mm} .{ }^{8} \mathrm{O}$ tempo de gelação é um conceito empregado em algumas áreas da indústria, mas que comumente deixa a desejar em termos de uma definição estrita a ser aplicada de forma ampla, de modo que existe uma arbitrariedade associada ao seu emprego. Desse modo, a determinação da grandeza a ser denominada tempo de gelação requereu uma convenção prévia que se adaptasse ao método aplicado para se estudar a mesma.

\section{EXPERIMENTAL}

\section{Preparação das amostras}

Os estudantes prepararam os componentes da amostra em duas composições distintas (dois níveis) com razões mássicas de gelatina e água destilada de 1:9 e 1:18, cada um deles correspondendo a um volume inicial de $40 \mathrm{~mL}$ de solução. Como cada ensaio requer condições distintas, a aleatorização dos mesmos foi necessária para distribuir igualmente a contribuição dos fatores desconhecidos sobre as respostas (de forma que se possam denominar essas contribuições de erros aleatórios) e os ensaios foram realizados um de cada vez, empregando os mesmos recipientes e instrumentos. A solução correspondente a cada ensaio foi preparada em um béquer de 250 $\mathrm{mL}$ imerso em outro béquer de $500 \mathrm{~mL}$, de forma a garantir uma temperatura estável nos valores desejados dentro de uma faixa de variação de aproximadamente $\pm 5^{\circ} \mathrm{C}$. A agitação foi proporcionada por um agitador magnético funcionando a um terço de sua velocidade de rotação máxima e o período de agitação para cada um dos ensaios foi de $10 \mathrm{~min}$. Após agitação, as emulsões produzidas foram levadas a um banho frio, à temperatura de resfriamento respectiva, e iniciaram-se as medições do tempo de gelação.

\section{Instrumentação (homemade)}

Um simples instrumento confeccionado pelos próprios estudantes para este estudo consiste de um prego invertido fixado em uma 
base de borracha e suportado por fios de algodão amarrados em uma garra metálica em um suporte universal, de forma a compor uma estrutura suspensiva e consideravelmente estável em termos de equilíbrio mecânico, passível de movimentação vertical. Sendo a massa do instrumento uma grandeza de valor fixo, o seu peso pode ser considerado um parâmetro invariável a ser empregado na convenção da metodologia de estudo do tempo de gelação. O peso aparente do aparato confeccionado fornece a força responsável pela imersão da base (superfície plana) do prego no gel de gelatina em água. O instante em que essa força já não seja suficiente para que toda a base do prego (com uma espessura aproximada de $2 \mathrm{~mm}$ ) possa imergir no gel (devido à resistência oferecida pela superfície da mesma) foi então definido como sendo o tempo de gelação. As medidas foram realizadas em intervalos de $15 \mathrm{~s}$. A Figura 1 apresenta um arranjo esquemático e uma fotografia mostrando o instrumento confeccionado.
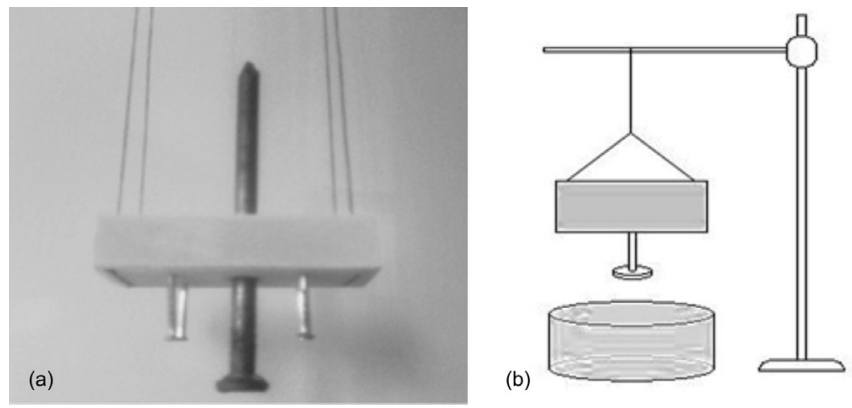

Figura 1. (a) Instrumentação desenvolvida pelos estudantes para determinação do tempo de pega; (b) representação esquemática do instrumento

\section{Planejamento fatorial completo $2^{3}$}

Foi aplicado um planejamento fatorial completo com 3 fatores (temperatura de dissolução da gelatina, temperatura de resfriamento e razão entre as massas de gelatina e água). Cada um dos fatores selecionados para manipulação foi escolhido de forma a ser variado em dois níveis. Os níveis foram escolhidos com base nas informações disponíveis sobre a influência dos fatores no comportamento da gelatina. Para a temperatura de dissolução, foram escolhidos os valores de 55 e $80^{\circ} \mathrm{C}$. Para a temperatura de resfriamento, foram escolhidos os valores de 4 e $14^{\circ} \mathrm{C}$. Para a razão mássica gelatina/água, os valores escolhidos foram 1:9 e 1:18.

As respostas coletadas para cada ensaio e suas respectivas réplicas foram tratadas com base em uma planilha em formato excel, contendo todos os algoritmos básicos do planejamento fatorial $2^{3}$. Por convenção, os valores numericamente maiores para cada um dos fatores foram adotados como os níveis superiores e aqueles numericamente menores, como inferiores. Desse modo, uma matriz de coeficientes de contraste, $\mathrm{X}$, foi montada associando o valor +1 aos níveis superiores e -1 aos níveis inferiores, de modo a dispor em colunas os níveis por fatores e os efeitos de interações (e uma coluna inicial adicional associada à média) e em linhas os ensaios realizados.

A matriz de coeficientes de contraste permite calcular, empregando álgebra matricial, os efeitos correspondentes a cada um dos fatores e suas interações, além da resposta média. Esses efeitos são obtidos ao se pré-multiplicar o vetor resposta, $\mathrm{R}$, pela transposta da matriz de coeficientes de contraste, seguindo-se pela divisão de cada componente do vetor produto, $\mathrm{p}$, por $2^{(\mathrm{n}-1)}$ e pela divisão adicional do primeiro termo por 2 - para um planejamento fatorial $2^{\mathrm{n}}$.

$$
p=X^{t} \otimes R
$$

Com esses valores determinados, resta obter uma estimativa do limiar de confiança a partir do qual se pode afirmar que os efeitos estudados são, de fato, importantes na determinação da resposta de interesse. Esse limiar é obtido pelo produto do parâmetro de Student respectivo $\left(t_{8}\right.$, nesse caso) a um dado intervalo de confiança (90, 95 ou $98 \%$ ) com o desvio padrão para um efeito $\left(\mathrm{s}_{(\text {efeito) }}\right)$. O desvio padrão de um efeito é obtido a partir da variância de um efeito, que por sua vez é determinada pela variância de um ensaio, s, calculada com base no produto interno do vetor diferença (d) e no número $n$ de fatores estudados $\left(n=3\right.$, nesse caso). Para uma análise fatorial $2^{3}$, a variância de um efeito é um quarto da variância de um ensaio. $\mathrm{O}$ produto $\mathrm{t}_{8} \cdot \mathrm{s}_{\text {(efeito) }}$ representa o valor mínimo a partir do qual os efeitos calculados são representativos na obtenção da resposta de interesse para o intervalo de confiança desejado. A Figura 1 mostra a respectiva matriz de coeficientes de contraste.

$$
s^{2}=\frac{d^{t} \otimes d}{2 \cdot 2^{n}}
$$

\section{RESULTADOS E DISCUSSÃO}

Utilizando-se das respostas coletadas nos ensaios realizados em duplicata e randomizados, os estudantes realizaram uma modelagem fatorial $2^{3}$. A Tabela 1 representa a matriz de planejamento fatorial para os ensaios. Para o cálculo dos efeitos e das interações no experimento, os estudantes escreveram as Equações 3 e 4 (em forma matricial, conforme previamente apresentado) em uma planilha Excel $^{\circledR}$ da Microsoft. $^{9}$

$$
\begin{gathered}
\langle y\rangle=\frac{\sum_{i=1}^{2 N} y_{i}}{2 N} \\
e f_{i}=\frac{\sum_{i=1}^{N} y_{i}^{+}-\sum_{k=1}^{N} y_{k}^{-}}{N}
\end{gathered}
$$

onde $N=2^{n}$ refere-se ao número de diferentes condições de ensaios (nesse caso, 8) e $y_{i}$ às observações individuais. A Equação 1 descreve a média de todas as observações, enquanto a Equação 2 descreve o cálculo do efeito para o j-ésimo fator ou interação, usando a diferença entre a soma das respostas dos ensaios em que o fator ou interação de interesse está no nível superior, +1 (representadas por $y_{i}^{+}$), e a soma das respostas em que o respectivo fator ou interação está no nível inferior, -1 (representadas por $y_{k}^{-}$). Vale notar que, no cálculo da média, um fator adicional de 2 é incluído

Tabela 1. Matriz de planejamento fatorial completo. A primeira coluna representa a ordem dos ensaios (a ordem de realização do primeiro ensaio seguido da ordem de realização da réplica)

\begin{tabular}{cccc}
\hline & \multicolumn{3}{c}{ Fatores } \\
\cline { 2 - 4 } Experimentos & $X_{1}$ & $X_{2}$ & $X_{3}$ \\
\cline { 2 - 4 } & $\begin{array}{c}\text { Temperatura de } \\
\text { resfriamento }\left({ }^{\circ} \mathrm{C}\right)\end{array}$ & $\begin{array}{c}\text { Temperatura de } \\
\text { dissolução }\left({ }^{\circ} \mathrm{C}\right)\end{array}$ & $\begin{array}{c}\text { Proporção } \\
\text { gelatina/água }\end{array}$ \\
\hline $1 \mid 2$ & 4 & 55 & $1: 18$ \\
$4 \mid 8$ & 14 & 55 & $1: 18$ \\
$2 \mid 6$ & 4 & 80 & $1: 18$ \\
$3 \mid 7$ & 14 & 80 & $1: 18$ \\
$8 \mid 1$ & 4 & 55 & $1: 9$ \\
$6 \mid 4$ & 14 & 55 & $1: 9$ \\
$7 \mid 3$ & 4 & 80 & $1: 9$ \\
$5 \mid 5$ & 14 & 80 & $1: 9$ \\
\hline
\end{tabular}


no denominador e no somatório em decorrência do fato de que todos os ensaios foram realizados em replicata. Como na Equação 2 apenas metade do número total de ensaios $(2 \cdot \mathrm{N})$ está incluso no cálculo de cada uma das médias a serem subtraídas, os somatórios e o denominador contém o número $\mathrm{N}$.

Os resultados obtidos para o cálculo dos efeitos entre os fatores investigados e seus respectivos efeitos foram colocados em forma de colunas, conforme mostrado na Figura 2. As linhas em horizontal correspondem aos intervalos de confiança (90, 95 e 98\%) calculados. Conforme mostrado na Figura 2, os valores absolutos mais pronunciados para os fatores de interesse foram temperatura de dissolução da gelatina e razão mássica. Para as interações entre dois fatores, o efeito mais pronunciado foi aquele relacionado à temperatura de dissolução (C), razão mássica (D) e, finalmente, o efeito da interação simultânea entre os três fatores foi de 1,5. Dependendo do nível de confiança desejado, os efeitos podem ser numericamente avaliados quanto à sua significância para a obtenção da resposta estudada. Para um nível de confiança de $90 \%$, apenas o efeito da interação entre a temperatura de resfriamento (B) e a temperatura de dissolução (C) é desconsiderável, sendo o único valor absoluto inferior ao limiar de significância de $0,930\left(\mathrm{~s}_{(\text {efeito) }} \cdot \mathrm{t}_{8(90 \%)}\right)$. Para um nível de confiança de $95 \%$, entretanto, a interação entre a temperatura de resfriamento (B) e a razão mássica (D) passa a ser também desconsiderável, por estar, em termos de valores absolutos, quantitativamente abaixo do limiar de 1,155 . Se o nível de confiança exigido for de $98 \%$, os únicos efeitos a se pronunciar serão o da razão mássica (D), da temperatura de dissolução (C), da interação entre os mesmos e da interação simultânea entre todos os fatores estudados, estando todos acima do limiar de confiança de 1,45 (Tabela 2).

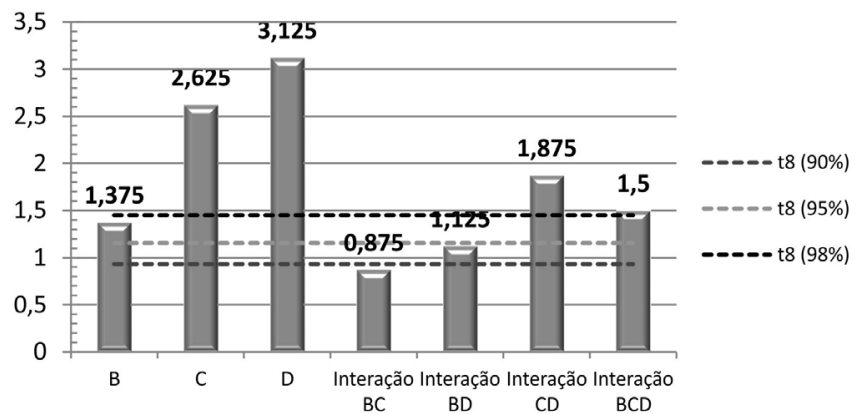

Figura 2. Gráfico de colunas para os efeitos calculados de cada um dos fatores. O comprimento vertical de cada coluna é proporcional à magnitude do efeito (cujo valor aparece acima da coluna respectiva). As linhas pontilhadas representam os limiares de significância $\left(s_{(\text {efeito })} \cdot t_{8}\right)$ para diferentes níveis de confiança (90, 95 e 98\%). B: temperatura de resfriamento; C: temperatura de dissolução; D: proporção gelatinalágua

A significância desses efeitos (a um nível de confiança de 98\%) conduz a uma necessidade de entendimento do que os mesmos representam em termos práticos. Apesar do fato de que os dados coletados na literatura indicam um maior incremento e força gel para gelatinas resfriadas a menores temperaturas durante intervalos de tempo iguais (3), os resultados aparentemente contraditórios com relação a esse efeito mascararam a real importância da temperatura de resfriamento sob condições padronizadas de gelação, uma vez que as medições foram realizadas sem padronização do tempo de resfriamento (já que o tempo era a resposta do experimento) e os ensaios tiveram graus diferentes de diluição da gelatina em água. Além disso, a variação na temperatura de resfriamento foi demasiadamente pequena para que efeitos pronunciáveis pudessem ser detectados. Segundo a literatura, ${ }^{6}$ o incremento percentual da força gel é mais pronunciado naqueles com menores concentrações
Tabela 2. Respostas coletadas para cada um dos ensaios. A média dos resultados é representada por $\langle Y\rangle$

\begin{tabular}{cccc}
\hline \multicolumn{4}{c}{ Respostas } \\
\hline \multirow{3}{*}{ Experimentos } & \multicolumn{3}{c}{ Tempo de pega (min) } \\
\cline { 2 - 4 } & $Y_{1}$ & $Y_{2}$ & $\langle Y\rangle$ \\
\hline $1 \mid 2$ & 14,25 & 11,25 & 12,75 \\
$4 \mid 8$ & 8,75 & 10,50 & 9,63 \\
$2 \mid 6$ & 15,00 & 15,25 & 15,13 \\
$3 \mid 7$ & 10,75 & 11,75 & 11,25 \\
$8 \mid 1$ & 11,25 & 10,00 & 10,63 \\
$6 \mid 4$ & 11,75 & 12,75 & 12,25 \\
$7 \mid 3$ & 18,50 & 18,00 & 18,25 \\
$5 \mid 5$ & 13,00 & 13,25 & 13,13 \\
\hline
\end{tabular}

de gelatina. Entretanto, os incrementos absolutos nos valores das forças géis são uma função crescente da razão mássica e géis mais concentrados devem apresentar maiores forças e menores tempos de gelação, o que está em concordância com o valor negativo para o efeito da razão mássica. Evidências experimentais revelam que menores temperaturas resultam em um processo de diluição mais eficiente, com pouca formação de aglomerados, enquanto que a temperaturas mais altas a dissolução tende a ocorrer rapidamente e com formação de grandes quantidades de aglomerados; entretanto, para o curto intervalo de tempo empregado no processo de dissolução, a velocidade de dissolução é o efeito preponderante, por implicar em maior concentração de gelatina em solução.

Uma vez que a temperatura de dissolução está intimamente relacionada com o processo de diluição da gelatina, é de se esperar que gelatinas diluídas em maiores proporções sejam capazes de percolar de forma mais rápida sob condições de resfriamento adequadas; sendo assim, gelatinas com maiores índices de diluição devem apresentar, em média, menores tempos de gelação, como evidenciado pelo valor negativo do efeito da temperatura de dissolução.

\section{CONCLUSÃO}

A aplicação de planejamentos fatoriais fornece uma ferramenta importante para o estudo e entendimento de experimentos conduzidos em laboratório. Por meio de sua utilização, foi possível aos alunos realizarem experimentos e analisarem seus resultados de forma a se atingir, do ponto de vista pedagógico, resultados superiores aos dos procedimentos padronizados das práticas laboratoriais realizadas no Instituto de Química da UFRN. As discussões ao longo deste experimento permitiram o entendimento de tópicos especiais em quimiometria, especialmente no estudo da formulação e aplicação de metodologias e técnicas quantitativas para análise de respostas de interesse. Além disso, foram elucidados efeitos importantes no comportamento de suspensões de gelatina em água, reforçando os conhecimentos previamente coletados nas bibliografias. O procedimento proposto mostrou-se adequado como prática experimental em uma disciplina de quimiometria para alunos do curso de Química. Este experimento pode ser executado em 2 semanas, desde a confecção do instrumento utilizado para medição do tempo de gelação à construção dos modelos de planejamento fatorial.

\section{MATERIAL SUPLEMENTAR}

Está disponível em http://quimicanova.sbq.org.br, em arquivo pdf, com acesso livre. 


\section{AGRADECIMENTOS}

À PROGRAD-UFRN, Instituto de Química da UFRN e aos estudantes que cursaram a disciplina de Quimiometria ofertada em 2011.1 nesta instituição.

\section{REFERÊNCIAS}

1. http://goldbook.iupac.org/CT06948.html, acessada em Fevereiro 2012.

2. Neves, A. C. O.; Soares, G. M.; Morais, S. C.; Costa, F. S. L.; Porto, D. L.; Lima, K. M. G.; J. Pharm. Biomed. Anal. 2012, 57, 115.

3. Lima, K. M. G.; Raimundo Jr., I. M.; Pimentel, M. F.; Sens. Actuators, B 2011, 160, 691 .
4. Guo, L.; Colby, R. H.; Lusigna, C. P.; Howe, A. M.; Macromolecules 2003, 36, 10009.

5. Al-Saidi, G.; Rahman, M. S.; Al-Alawi, A.; Guizani, N.; J. Therm. Anal. Calorim. 2011, 104, 593.

6. Kravtchenko, T. P.; Parker, A.; Brigand, G.; Food Hydrocolloids 1999, $13,219$.

7. Larsen, C. K.; Gaserød, O.; Smidsrød, O.; Carbohydr. Polym. 2003, 51, 125.

8. Carpenter, D. C.; Dahlberg, A. C.; Hening, J. C.; Ind. Eng. Chem. Res. 1928, 20, 397.

9. http://www.microsoft.com, acessada em Março 2012. 